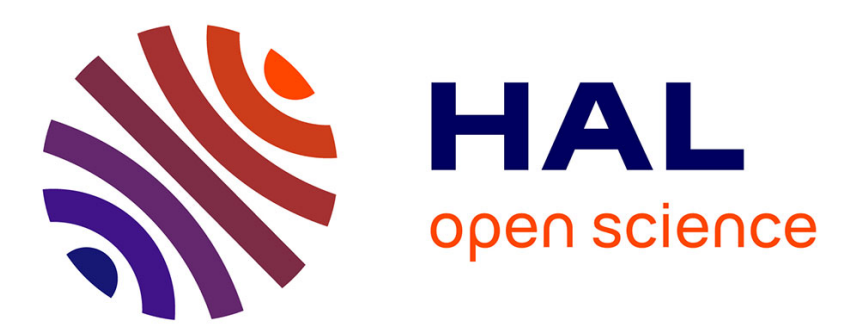

\title{
Modèle analytique du MESFET AsGa pour simulation de circuits logiques ultra-rapides
}

\author{
Laurent Chusseau, P. Crozat, R. Adde
}

\section{To cite this version:}

Laurent Chusseau, P. Crozat, R. Adde. Modèle analytique du MESFET AsGa pour simulation de circuits logiques ultra-rapides. Revue de Physique Appliquée, 1987, 22 (11), pp.1515-1527. 10.1051/rphysap:0198700220110151500 . jpa-00245704

\section{HAL Id: jpa-00245704 https://hal.science/jpa-00245704}

Submitted on 1 Jan 1987

HAL is a multi-disciplinary open access archive for the deposit and dissemination of scientific research documents, whether they are published or not. The documents may come from teaching and research institutions in France or abroad, or from public or private research centers.
L'archive ouverte pluridisciplinaire HAL, est destinée au dépôt et à la diffusion de documents scientifiques de niveau recherche, publiés ou non, émanant des établissements d'enseignement et de recherche français ou étrangers, des laboratoires publics ou privés. 


\title{
Modèle analytique du MESFET AsGa pour simulation de circuits logiques ultra-rapides
}

\author{
L. Chusseau, P. Crozat et R. Adde \\ Institut d'Electronique Fondamentale, Laboratoire associé au CNRS UA22, Bâtiment 220, Université Paris- \\ Sud, 91405 Orsay Cedex, France
}

(Reçu le 23 janvier 1987, révisé le 6 juillet 1987, accepté le 6 juillet 1987)

\begin{abstract}
Résumé. - Un modèle de MESFET AsGa est développé pour la conception des circuits logiques picosecondes à moyenne et grande intégration. La formulation des paramètres du modèle est optimisée à la fois pour un bon comportement dynamique des portes logiques et pour une grande efficacité en temps de calcul. Ce modèle est implanté sur le macro-simulateur MACPRO qui a été conçu pour l'étude des effets de propagation et de couplage dans les circuits intégrés ultrarapides. Le modèle est présenté de façon exhaustive et il est comparé à des mesures statiques (caractéristiques $I-V$ de transistors hyperfréquence) et dynamiques (comportement en paramètres $S$ d'un MESFET hyperfréquence, oscillateur en anneau en technologie BFL). La dernière partie de l'article étudie l'influence des paramètres importants de ce modèle sur le régime transitoire des portes logiques BFL.
\end{abstract}

\begin{abstract}
A model of the GaAs MESFET is developped for the design of picosecond circuits at the MSI or LSI level. The parameter description is optimized both for dynamic response and computer time efficiency. The model is implemented in the time simulator MACPRO designed for the analysis of propagation and coupling effects in very fast integrated circuits. The model is fully presented and validated against both experimental $I-V$ curves and microwave $S$ parameters measurements of a single MESFET, and analog dynamic response of a BFL ring oscillator. The influence of the main model parameters on the circuit dynamic response is discussed relatively to a BFL technology.
\end{abstract}

Liste des notations.

$q=-1.6 \times 10^{-19} \mathrm{C}$ charge élémentaire de l'électron

$\varepsilon=\varepsilon_{\mathrm{r}} \cdot \varepsilon_{0}$

\section{$k$}

$\mathbf{N}_{\mathbf{d}}$

$V_{\mathrm{bi}}=\phi_{\mathrm{s}}$

$V_{\mathrm{t}}^{\mathrm{bi}}$

$V_{\mathrm{p}}=V_{\mathrm{bi}}-V_{\mathrm{t}}$

$V_{\text {ds }}$

$V_{\text {gs }}$

$V_{\text {gd }}$

$I_{\mathrm{d}}$

$I_{\mathrm{dss}}$

$\mu_{0}$

Ce travail a été financé en partie par le contrat CNET $\mathrm{n}^{\circ} 848 \mathrm{~B} 006$. transconductance

largeur de grille

longueur de grille distance source-grille distance grille-drain distance source-drain longueur moyenne des métallisations de drain et de source température profondeur effective du canal pour un dopage uniforme conductance de drain constante décrivant la conductance de drain en fonction de $V_{\text {gs }}$

résistance carrée de la métallisation de grille

facteur de contact

hauteur de barrière entre l'AsGa et l'air facteur d'idéalité des diodes Schottky 


\section{Introduction.}

Depuis le début des années 1980, la conception de circuits intégrés monolithiques hyperfréquences (MMIC) a conduit au développement de modèles précis pour le MESFET AsGa [1-5]. Pour une telle application, les performances en temps calcul du modèle n'ont que peu d'importance puisque les MMIC ne font intervenir qu'un petit nombre de transistors. Par suite, les expressions semi-empiriques décrivant, soit la source de courant interne du MESFET AsGa, soit les dépendances non linéaires des éléments parasites, sont généralement complexes afin de conserver une grande précision [69]. De tels modèles ne peuvent être utilisés pour des applications de type circuits logiques correspondant à des niveaux d'intégration moyen (MSI) ou grand (LSI). De plus les programmes de simulation qui leur correspondent et qui permettent d'effectuer des simulations harmoniques ou grand signal sont la plupart du temps strictement privés. Il faut aussi souligner que l'implantation d'un modèle MESFET AsGa dérivé du modèle de Curtice dans un simulateur «public » comme SPICE est très récente [1012].

A l'opposé, des modèles très simplifiés de MESFET AsGa existent pour la simulation de circuits logiques [13]. La complexité usuellement admise pour les éléments non linéaires est assez faible, moyennant quoi la simulation de circuits comportant un grand nombre de portes logiques devient possible avec des coûts en temps machine assez faibles. D'une façon générale ces modèles sont une adaptation des équations du JFET silicium et la précision qu'il est possible d'obtenir est trop faible pour évaluer très finement les performances des circuits.

Les progrès de rapidité et de niveau d'intégration des circuits logiques AsGa créent un besoin de modèles de MESFET précis et bien adaptés à la simulation de circuits rapides complexes. Un compromis s'impose alors entre une représentation du comportement dynamique des portes logiques la plus fidèle possible et un niveau de complexité compatible avec des temps de calcul assez courts. Afin d'étudier les perturbations apportées par la propagation, le couplage entre lignes sur la puce, et le bruit de commutation dans les circuits logiques AsGa [14], nous avons développé un modèle de ce type.

La première partie de cet article présente le modèle dans son ensemble avec les équations des différents éléments. Des validations expérimentales sont proposées pour appuyer la formulation de certains éléments. La seconde partie concerne la comparaison entre des simulations et des expériences mettant en jeu le caractère dynamique du modèle (paramètres $S$ d'un MESFET hyperfréquence, oscillateur en anneau). Enfin la dernière partie propose une étude de sensibilité de la réponse dynamique des portes logiques BFL vis-à-vis des paramètres principaux du modèle.

\section{Le modèle de MESFET AsGa.}

2.1 REPRÉSENTATION ElEctrique DU MESFET AsGa. - La structure d'un MESFET AsGa est donnée en coupe figure 1. Les éléments parasites sont représentés sur cette coupe à l'endroit correspondant à leur localisation physique. Le tableau I résume l'importance de chacun d'eux dans le cadre de la simulation des circuits intégrés AsGa logiques. Parmi ces quinze éléments, six d'entre eux seulement proviennent du transistor intrinsèque (la zone de charge d'espace et le canal). Les autres éléments sont liés aux métallisations et aux contacts électriques nécessaires aux interconnexions. Une analyse théorique du dispositif montre que chaque élément issu du transistor intrinsèque $a$ un comportement non linéaire par rapport à deux au moins des trois tensions internes du FET. Nous avons choisi des formulations simplifiées de certains d'entre eux afin d'assurer l'objectif de faible coût CPU : $I_{\mathrm{d}}, C_{\mathrm{gs}}, C_{\mathrm{gd}}$ et $D 1$ sont effectivement décrits en utilisant des expressions non linéaires simples, la résistance de canal $R_{\mathrm{i}}$ sera pour sa part choisie constante en fonction de la polarisation.

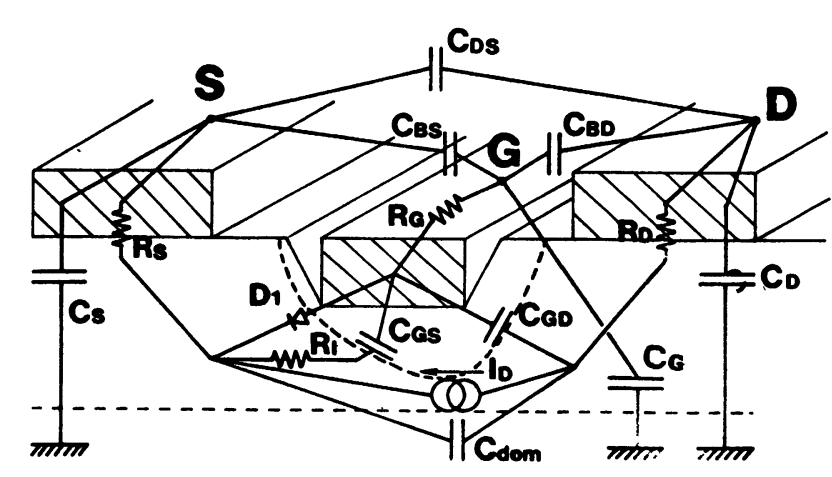

Fig. 1. - Vue en coupe d'un MESFET AsGa. Les éléments du FET intrinsèque et extrinsèque sont localisés à l'endroit de leur action physique.

[Cross-sectionnal view of a MESFET with recessed gate. Elements of both intrinsic and extrinsic FET are located within the device.]

La topologie du modèle physico-électrique équivalent est donnée figure 2. Les éléments de charge des portes BFL et DCFL (transistors montés en source de courant) voient fréquemment une inversion de polarité $\left(V_{\mathrm{ds}}<0 \mathrm{~V}\right)$ lorsque la porte commute. Il est alors très important de rendre compte du comportement réel de cette charge active (fonctionnement en inverse) afin de conserver la convergence de la résolution numérique du simulateur. Le moyen 
Tableau I. - Importance et origine physique des éléments de la figure 1. L'importance donnée est relative à la simulation temporelle de circuits logiques ultrarapides. Les remarques donnent succinctement l'influence de chaque élément sur la réponse transitoire des MESFET.

[Importance and physical origin of the elements of figure 1. The importance is given relatively to ultrafast logic circuit simulation. The remarks are related to the element influence on the MESFET response.]

\begin{tabular}{|c|c|c|}
\hline & Origine Physique & Importance: Influence de l'Eléme \\
\hline \multicolumn{3}{|c|}{ *** FET Intrinseque *** } \\
\hline${ }_{\mathrm{R}_{i}}{ }_{\mathrm{D}_{i}}{ }^{\cdots}$ & 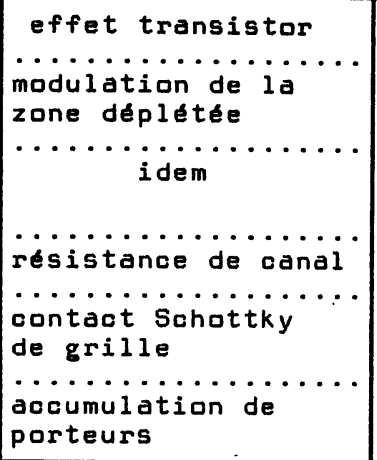 & 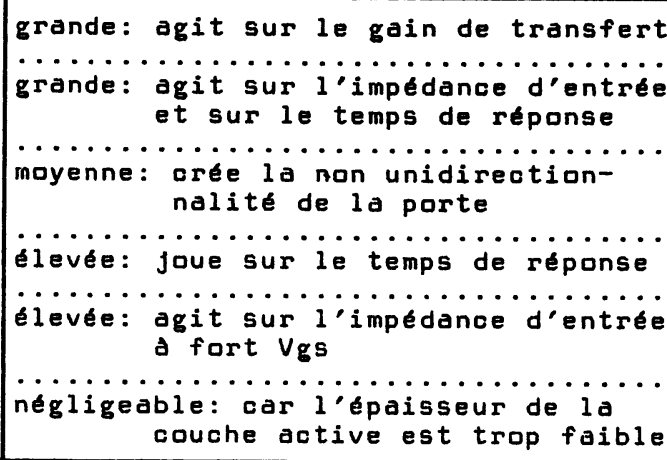 \\
\hline \multicolumn{3}{|c|}{ *** FET Extrinseque *** } \\
\hline 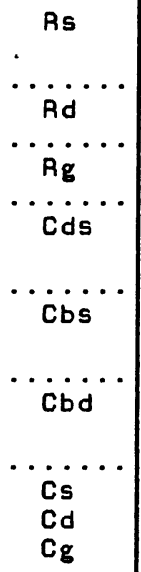 & 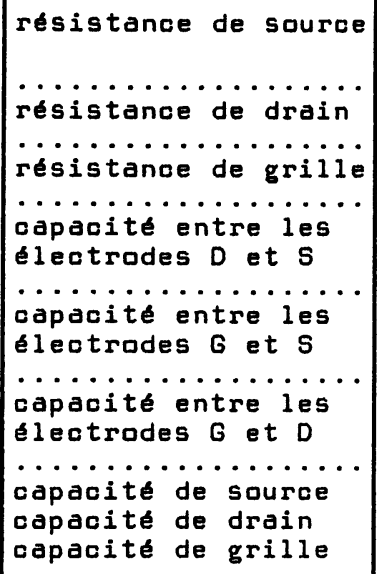 & 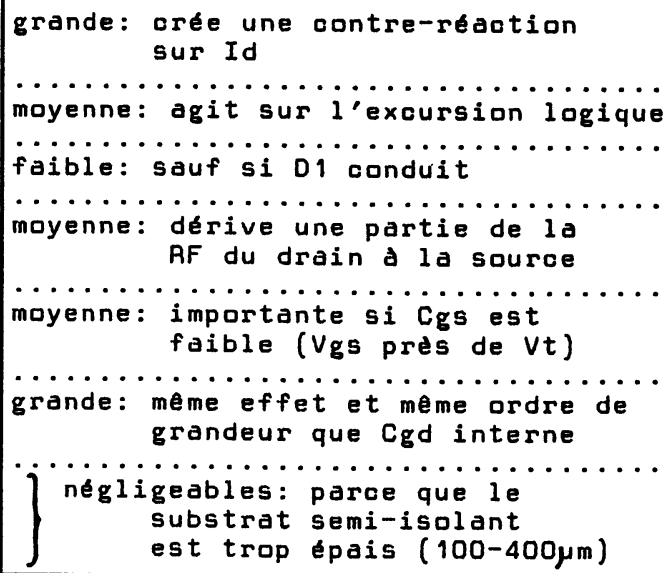 \\
\hline
\end{tabular}

choisi est de symétriser totalement le schéma physico-électrique équivalent du MESFET, ce qui fait jouer des rôles identiques aux nœuds source et drain du transistor. Cela complique légèrement le schéma électrique et la figure 2 diffère ainsi du schéma généralement choisi pour la modélisation de ces dispositifs en hyperfréquence. Une comparaison directe des éléments entre les différents types de modèles est impossible.

La source de courant interne du modèle présenté possède une dépendance non linéaire avec les deux tensions $V_{\mathrm{gs}}^{\prime}=V_{\mathrm{g}}^{\prime}-V_{\mathrm{s} 2}$ et $V_{\mathrm{d}}^{\prime}-V_{\mathrm{s}}^{\prime}$ qui représentent respectivement la différence de potentiel aux bornes de la zone de charge d'espace et la différence de potentiel interne entre l'extrémité drain et l'extrémité source du canal. La dépendance $I_{\mathrm{d}}=f\left(V_{\mathrm{gs}}^{\prime}\right)$ introduit un temps de réponse à la modification de la forme de la zone de charge d'espace à travers la conjonction des effets de $R_{\mathrm{i}}$, résistance de canal et de
$C_{\mathrm{gs}}$, capacité grille source du FET intrinsèque. Les éléments capacitifs non linéaires $C_{\mathrm{gs}}$ et $C_{\mathrm{gd}}$ dépendent respectivement de $V_{\mathrm{gs}}^{\prime}$ et de $\left(V_{\mathrm{d}}^{\prime}-V_{\mathrm{d} 2}\right)$ alors que le courant des diodes Schottky $D 1$ et $D 2$ est lié à $\left(V_{\mathrm{g}}^{\prime}-V_{\mathrm{s}}^{\prime}\right)$ et $\left(V_{\mathrm{g}}^{\prime}-V_{\mathrm{d}}^{\prime}\right)$ respectivement.

La suite du texte discute chacun des éléments du modèle séparément, une partie d'entre eux sera discuté en s'appuyant sur des validations expérimentales concernant trois technologies submicroniques différentes (voir Tab. II). Les paramètres relatifs au NEC388 ont été fournis par Cappy dans sa thèse [15]. Le transistor Thomson 1408 a été construit sur une plaquette épitaxiée par jet moléculaire alors que la technologie utilisée pour la réalisation de circuit BFL utilise l'implantation ionique.

\subsection{PARAMÈTRES DU TRANSISTOR INTRINSÈQUE.}

2.2.1 Caractéristique statique I-V. - La modélisation de la caractéristique statique $I-V$ fait appel à la 


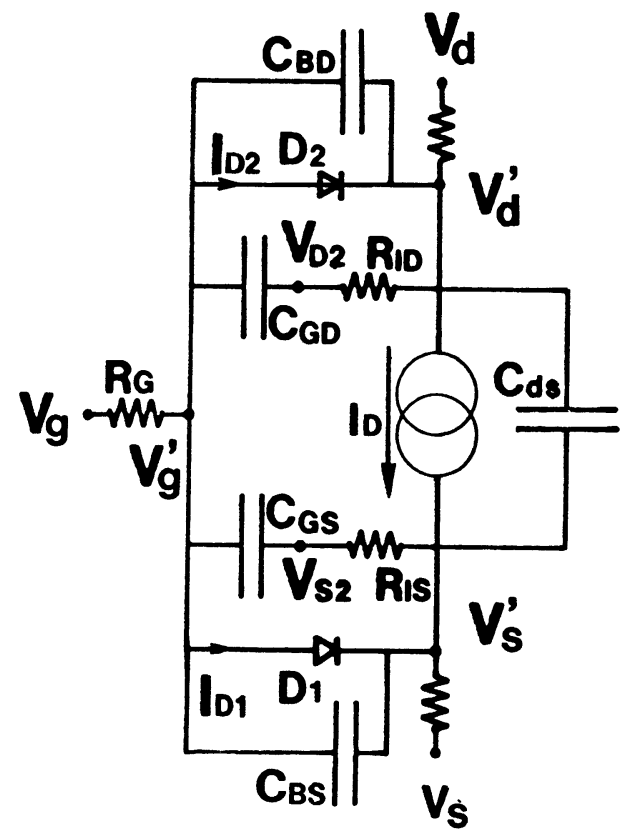

Fig. 2. - Topologie symétrisée du schéma équivalent du MESFET. Les éléments non linéaires sont la source de courant $I_{\mathrm{d}}$, les capacités grille-source $C_{\mathrm{gs}}$ et grille-drain $C_{\text {gd }}$ et les diodes Schottky $D 1$ et $D 2$. Les tensions de commande sont: $\left(V_{\mathrm{g}}^{\prime}-V_{\mathrm{s} 2}\right)$ et $\left(V_{\mathrm{d}}^{\prime}-V_{\mathrm{s}}^{\prime}\right)$ pour $I_{\mathrm{d}}$, $\left(V_{\mathrm{g}}^{\prime}-V_{\mathrm{s} 2}\right)$ pour $C_{\mathrm{gs}},\left(V_{\mathrm{g}}^{\prime}-V_{\mathrm{d} 2}\right)$ pour $C_{\mathrm{gd}},\left(V_{\mathrm{g}}^{\prime}-V_{\mathrm{s}}^{\prime}\right)$ pour $D 1,\left(V_{\mathrm{g}}^{\prime}-V_{\mathrm{d}}^{\prime}\right)$ pour $D 2$.

[Symmetrical circuit schematic of the MESFET. Nonlinear elements are the channel current source $I_{\mathrm{d}}$, the gatesource $C_{\mathrm{gs}}$ and gate-drain $C_{\mathrm{gd}}$ internal capacitances, and the Schottky diodes $D 1$ and $D 2$. Command voltages are : $\left(V_{\mathrm{g}}^{\prime}-V_{\mathrm{s} 2}\right)$ and $\left(V_{\mathrm{d}}^{\prime}-V_{\mathrm{s}}^{\prime}\right)$ for $I_{\mathrm{d}},\left(V_{\mathrm{g}}^{\prime}-V_{\mathrm{s} 2}\right)$ for $C_{\mathrm{gs}}$, $\left(V_{\mathrm{g}}^{\prime}-V_{\mathrm{d} 2}\right)$ for $C_{\mathrm{gd}},\left(V_{\mathrm{g}}^{\prime}-V_{\mathrm{s}}^{\prime}\right)$ for $D 1,\left(V_{\mathrm{g}}^{\prime}-V_{\mathrm{d}}^{\prime}\right)$ for D2.]

théorie uni-dimensionnelle du JFET établie par Shockley [16]. Nous avons défini un critère supplémentaire afin de tenir compte de la saturation de vitesse des porteurs qui est le principal mode de saturation du courant dans les MESFET submicroniques AsGa.

Le comportement du canal sous la grille en régime de saturation de courant peut être séparé en deux régions distinctes (Fig. 3) qui sont caractérisées de la façon suivante en utilisant un modèle de mobilité par segment :

(i) Une région validant les hypothèses de Schockley où les porteurs possèdent la valeur de mobilité en champ faible $\mu_{0}$.

(ii) Une région où les porteurs ont la vitesse de dérive de saturation.

La dépendance par segment de la mobilité permet la simplification suivante : la vitesse de dérive des électrons sature [17] lorsqu'ils ont été accélérés sous une différence de potentiel interne entre source et drain d'environ 0,4 V. Ainsi l'énergie propre d'un électron du canal ne peut être inférieure à $0,4 \mathrm{eV}$
Tableau II. - Paramètres technologiques et géométriques des transistors hyperfréquences NEC388 et TH1408 utilisés pour des validations expérimentales du modèle. Les paramètres typiques des technologies BFL projetées et réalisées par THOMSON DHM sont aussi donnés.

[Technological and geometrical parameters of NEC388 and THOMSON 1408 microwave MESFETs used for model validation. Typical values of THOMSON DHM BFL in the dynamic comparisons are also given for both realized and projected technologies.]

\begin{tabular}{|c|c|c|c|c|c|}
\hline Parametre & Unité & NEC388 & TH1408 & $\begin{array}{c}\text { BFL } \\
\text { réalisée }\end{array}$ & $\begin{array}{c}B F L \\
\text { projetée }\end{array}$ \\
\hline $1 \mathrm{~g}$ & $\mu \mathrm{m}$ & 0.55 & 0.5 & 1 & 0.6 \\
\hline w & $\underset{\mu m}{m}$ & ${ }_{280} \cdots$ & 260 & $\cdots_{10} \cdots$ & $\cdots \cdots_{20} \cdots$ \\
\hline$\cdots_{v_{b i}} \cdots$ & $\cdots$ & $\cdots_{0.7} \cdot$ & $\ddot{0.8} \cdot$ & $\ddot{0.7}^{\prime} \cdots$ & $\cdots_{0 . j} \cdots$ \\
\hline $\mathrm{gc}$ & $\dot{s} / \dot{v} / \mu \mathrm{m}$ & $\cdots_{10} \cdots$ & $\ddot{6.1} \cdots$ & ${ }_{\theta} \cdots \cdots$ & 13 \\
\hline$\mu_{0}$ & $\mathrm{~cm}^{2} / \dot{v} / \dot{s}$ & ${ }_{2000}$ & 2250 & 2500 & 2500 \\
\hline$\cdots \cdots$ & $\ddot{\mu m}_{m} \cdot$ & $\cdots_{0.12} \cdots$ & $\ddot{0.16}$ & $0.12 \cdots$ & $\cdots \cdots_{0.12}$ \\
\hline$\ddot{N}$ & $\because \cdots$ & $\cdots_{1.2} \cdots$ & $\cdots \ddot{1.2} \cdots$ & $\cdots_{1.2} \cdots$ & $\ddot{1.2}$ \\
\hline$\ddot{R g}$ & & $\cdots \cdots$ & ${ }_{5.8} \cdots$ & $\ddot{5}$ & $\cdots_{16.7}$ \\
\hline$\ddot{R d}$ & & $\cdots_{10.3} \cdots$ & $\cdots \cdots$ & $\cdots_{104} \cdots$ & $\cdots_{46.7}$ \\
\hline$\ddot{\text { Ris }}$ & & ${ }_{10.7}$ & $\ddot{3.9} \cdots$ & $\operatorname{mid}_{104} \cdots$ & $\cdots_{46 . j}$ \\
\hline$\ddot{\mathrm{Nd}}$ & $\ddot{A t} / \mathrm{cm}^{3}$ & $\ddot{2.2 E} \dot{2}$ & $\ddot{1.8 \mathrm{E} i{ }^{\prime}}$ & $\dot{2 E}_{17} \cdots$ & 2E 17 \\
\hline isg & $\cdots \cdots$ & $\ddot{0 . \theta} \cdots$ & ${ }_{0.9} \cdots$ & $\cdots \dddot{i . s} \cdots$ & $\ddot{0} \dot{8}$ \\
\hline isd & $\cdots$ & ${ }_{2.5} \cdots$ & $3.5 \cdots$ & $\ddot{4} \cdots$ & $\ddot{2} 2$ \\
\hline$\ddot{L}$ & $\cdots \cdots$ & $\cdots_{50} \cdots$ & $\cdots$ & 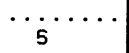 & $\cdots_{5} \cdots$ \\
\hline
\end{tabular}

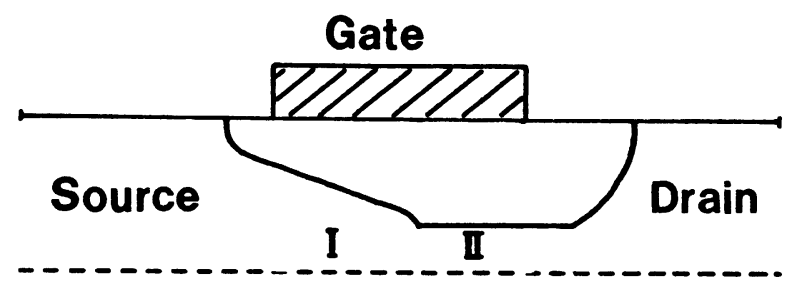

Fig. 3. - Zone de déplétion sous la grille en régime de saturation. Deux régions sont à distinguer : (I) la région validant les hypothèses de Shockley, (II) la région où les porteurs ont la vitesse de dérive de saturation.

[Depleted region under the gate in saturation regime. Two different regions are distinguished: (I) the Shockley region, (II) the region where carriers have the saturated drift velocity.]

[18]. Cette condition apparaît très raisonnable dans le cas des dispositifs à grille courte, $l_{\mathrm{g}}$ doit cependant rester suffisamment grand $\left(l_{\mathrm{g}}>0,3 \mu \mathrm{m}\right)$ pour que le comportement balistique des porteurs reste négligeable.

Avec cette hypothèse la modélisation que nous utilisons est identique à celle de Shockley tant que 
$V_{\mathrm{ds}}<0,4 \mathrm{~V}$ (conditions (b) et (c) ci-dessous). Dès que la tension interne aux bornes du canal dépasse $0,4 \mathrm{~V}$, le mécanisme de saturation du courant est la saturation de mobilité, sauf si $\left(V_{\mathrm{gs}}^{\prime}-V_{\mathrm{t}}\right)<0,4 \mathrm{~V}$

$$
\begin{aligned}
& \left(V_{\mathrm{gs}}^{\prime}-V_{\mathrm{t}}+0,15 \mathrm{~V}\right)<0 \\
& \left(V_{\mathrm{gs}}^{\prime}-V_{\mathrm{t}}\right)<V_{\mathrm{ds}} \\
& \left(V_{\mathrm{gs}}^{\prime}-V_{\mathrm{t}}\right)>V_{\mathrm{ds}} \\
& \left(V_{\mathrm{gs}}^{\prime}-V_{\mathrm{t}}\right)<0,4 \mathrm{~V} \\
& \left(V_{\mathrm{gs}}^{\prime}-V_{\mathrm{t}}\right)>0,4 \mathrm{~V}
\end{aligned}
$$

Où :

$$
\begin{gathered}
I_{\mathrm{d} 1}=2 \cdot \beta \cdot\left\{\left(V_{\mathrm{gs}}^{\prime}-V_{\mathrm{t}}\right) \cdot \tilde{V}_{\mathrm{ds}}-\frac{\tilde{V}_{\mathrm{ds}}^{2}}{2}\right\}+g_{\mathrm{d}} \cdot V_{\mathrm{ds}} \\
I_{\mathrm{d} 2}=\beta \cdot\left(V_{\mathrm{gs}}^{\prime}-V_{\mathrm{t}}\right)^{2}+g_{\mathrm{d}} \cdot V_{\mathrm{ds}}
\end{gathered}
$$

$\tilde{V}_{\mathrm{ds}}$ représente la tension d'accélération des porteurs sous le canal, par conséquent elle inclue la saturation et sa valeur est le minimum de $V_{\text {ds }}$ et de $0,4 \mathrm{~V}$

$$
\begin{gathered}
V_{\mathrm{t}}=\frac{q \cdot N_{\mathrm{d}} \cdot a_{0}^{2}}{2 \cdot \varepsilon}+V_{\mathrm{bi}} \\
\beta=\frac{w}{l_{\mathrm{g}}} \cdot \frac{\mu_{0} \cdot \varepsilon}{2 \cdot a_{0}}
\end{gathered}
$$

$V_{\mathrm{t}}$ et $\beta$ suivent exactement la formulation de Shockley, ils dépendent de façon directe de $a_{0}$ et de $\mu_{0}$ qui sont deux paramètres difficiles à mesurer précisément.

(i) La méthode la plus courante aujourd'hui de fabrication des MESFET utilise l'implantation ionique ; il en découle que la frontière entre la couche active et le substrat semi-isolant n'est pas très bien définie. Au vu des profils de dopages existant dans la littérature la profondeur de la couche active $a_{0}$ s'étend typiquement sur une distance de $200 \AA$ conjointement avec la décroissance du dopage. Cette incertitude peut être utilisée dans le présent modèle pour ajuster la tension de seuil en utilisant l'équation (3).

(ii) Il a été montré que la mobilité en champ faible sous la grille des MESFET possède des valeurs nettement plus faibles que celles mesurables par une technique d'effet Hall [19]. Dans le cas d'un MESFET implanté de longueur de grille $1 \mu \mathrm{m}$ la mobilité en champ faible a été mesurée dans la gamme $2000-2500 \mathrm{~cm}^{2} / \mathrm{V} \cdot \mathrm{s}$ selon la profondeur considérée sous la grille. Pour la plaquette ayant donné naissance à ce dispositif la mesure de mobilité par effet Hall donnait $3000-4000 \mathrm{~cm}^{2} / \mathrm{V} \cdot \mathrm{s}$. Cette remarque car alors une saturation classique de type JFET existe déjà (conditions (d) et (e) ci-dessous).

L'ensemble de la caractéristique est décrit par les 5 conditions :

$$
\begin{array}{ll} 
& I_{\mathrm{d}}=0 \\
V_{\mathrm{ds}}<0,4 \mathrm{~V} & I_{\mathrm{d}}=I_{\mathrm{d} 2} \\
V_{\mathrm{ds}}<0,4 \mathrm{~V} & I_{\mathrm{d}}=I_{\mathrm{d} 1} \\
V_{\mathrm{ds}}>0,4 \mathrm{~V} & I_{\mathrm{d}}=I_{\mathrm{d} 2} \\
V_{\mathrm{ds}}>0,4 \mathrm{~V} & I_{\mathrm{d}}=I_{\mathrm{d} 1} .
\end{array}
$$

explique les valeurs de $\mu_{0}$ tableau II qui sont assez inhabituelles pour de l'arséniure de gallium massif [17]. De même que l'incertitude sur $a_{0}$, l'incertitude sur $\mu_{0}$ peut être mise à profit pour ajuster la caractéristique $I-V$ d'un transistor à une mesure.

L'origine physique de la conductance de drain des MESFET se trouve dans l'injection de porteurs dans le substrat semi-isolant générée par la composante perpendiculaire du champ électrique dans le canal. Puisque la frontière entre canal et substrat n'est pas abrupte dans le dispositif réel, cette conductance de drain est extrêmement difficile à modéliser sans l'aide d'analyses bi-dimensionnelles ou particulaires appliquées à chaque cas de profil de dopage. Nous avons donc choisi d'utiliser une formule semi-empirique issue de ces simulations bi-dimensionnelles ou particulaires [20-24]

$$
g_{\mathrm{d}}=w \cdot g_{\mathrm{c}} \cdot\left(V_{\mathrm{gs}}^{\prime}-V_{\mathrm{t}}+0,15 \mathrm{~V}\right)
$$

Les équations (1) et (2) vérifient la continuité de $I_{\mathrm{d}}$ au passage du fonctionnement en régime non saturé au fonctionnement en régime saturé. Cependant la dérivée première de $I_{\mathrm{d}}$ ne vérifie pas cette condition. Dans le cas de la simulation de circuits logiques les transistors sont utilisés pour leurs propriétés de commutation, ainsi les parties les plus importantes de la caractéristique $I-V$ sont celles mettant en jeu la saturation et le blocage que nous nous sommes attachés à modéliser correctement.

Les figures 4 et 5 montrent des comparaisons simulation-mesure de caractéristiques $I-V$ relatives aux transistors hyperfréquences dont les paramètres sont donnés tableau II. Dans le cas du NEC388, une légère surévaluation du courant drain-source simulé est observable, cela est probablement la conséquence d'un mauvais choix de $\mu_{0}$.

Les tensions $V_{\mathrm{gs}}^{\prime}$ et $V_{\mathrm{ds}}$ sont internes au modèle, par conséquent les chutes de tensions aux bornes des résistances d'accès $R_{\mathrm{s}}$ et $R_{\mathrm{d}}$ font croître la tension drain-source externe où la saturation du courant apparaît. Ce phénomène est très visible sur les figures 4 et 5 où par exemple le passage du fonction- 


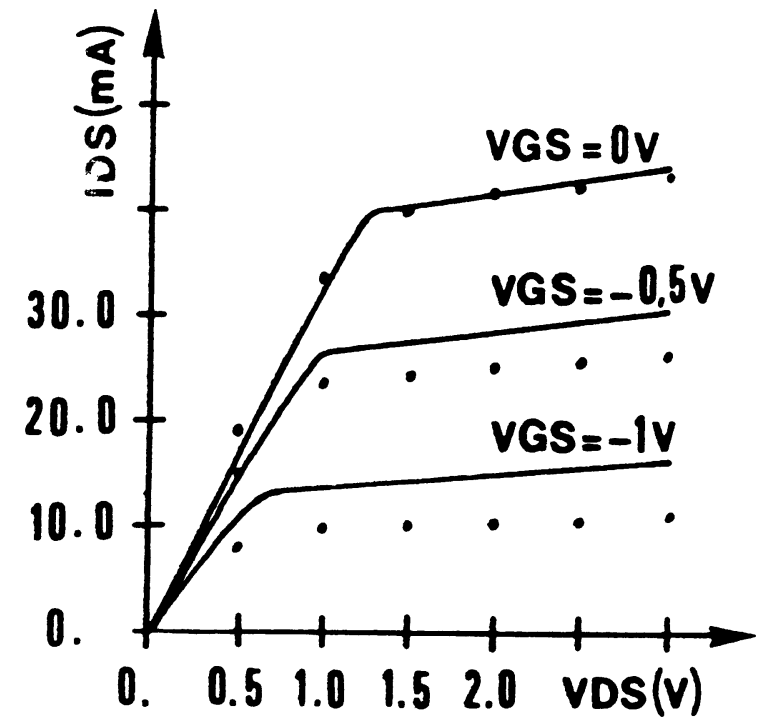

Fig. 4. - Comparaison des caractéristiques $I_{\mathrm{ds}}\left(V_{\mathrm{gs}}, V_{\mathrm{ds}}\right)$ externes pour le transistor NEC388. —_ résultats de simulation, ...... valeurs expérimentales.

[Static $I_{\mathrm{ds}}\left(V_{\mathrm{ds}}, V_{\mathrm{gs}}\right)$ external characteristic of the NEC388 microwave MESFET. - simulation results, ...... experimental measurements.]

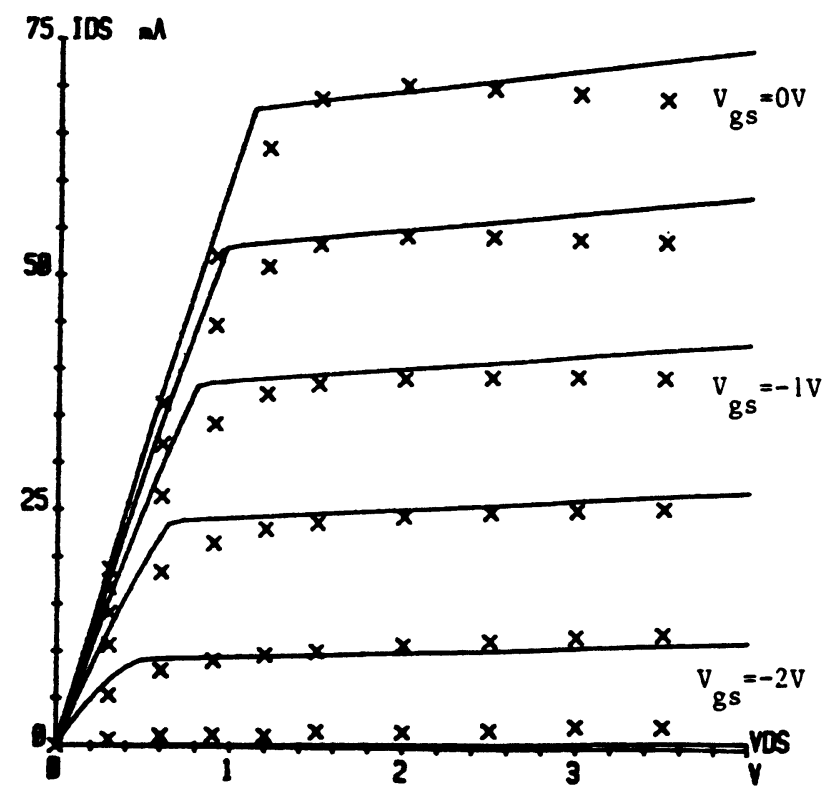

Fig. 5. - Comparaison des caractéristiques $I_{\mathrm{ds}}\left(V_{\mathrm{gs}}, V_{\mathrm{ds}}\right)$ externes pour le transistor TH1408. - résultats de simulation, $\times \times \times$ valeurs expérimentales.

[Static $I_{\mathrm{ds}}\left(V_{\mathrm{ds}}, V_{\mathrm{gs}}\right)$ external characteristic of the TH1408 microwave MESFET. - simulation results, $x \times \times$ experimental measurements.]

nement linéaire au fonctionnement saturé apparaît pour une tension drain-source externe de $\sim 1 \mathrm{~V}$.

Dans le cas du MESFET TH1408, la comparaison modèle-expérience est très satisfaisante (Fig. 5), la raison devant être trouvée dans la précision des informations concernant le processus de fabrication technologique. La seule divergence notable apparaît dans la conductance de drain qui croît avec $V_{\mathrm{gs}}^{\prime}$ dans le modèle, alors que les mesures montrent une décroissance lorsque $V_{\mathrm{gs}}>-0,5 \mathrm{~V}$. Cet effet observé expérimentalement correspond au phénomène bien connu de l'influence de la température dans le canal qui croît lorsque les densités de courant deviennent importantes, notamment près de $I_{\text {dss }}$.

Ces validations expérimentales sont l'occasion de noter que les discontinuités du premier ordre des caractéristiques internes du modèle sont très atténuées lorsque l'ensemble des éléments parasites est pris en compte, tout particulièrement les résistances d'accès (Figs. 4 et 5).

2.2.2 Capacités $d u$ transistor intrinsèque. - La dépendance non linéaire des capacités grille-source et grille-drain est donnée par des formulations analytiques correspondant aux différentes conditions physiques de fonctionnement : le régime avant pincement du canal, le régime après pincement du canal, une zone de transition assurant la continuité entre les régimes précédents. La modélisation choisie a été publiée antérieurement [25], et sa validité a été prouvée à la fois pour $C_{\mathrm{gs}}$ et $C_{\mathrm{gd}}$. Les équations (6) et (7) décrivent les variations de $C_{\mathrm{gs}}$ en fonction de la différence de tension appliquée à ses bornes.

Avant pincement du canal $\left(V_{\mathrm{gs}}^{\prime}>V_{\mathrm{t}}+0,15 \mathrm{~V}\right.$.)

$$
C_{\mathrm{gs}}=\frac{\pi}{2} \cdot \varepsilon w+\frac{w \cdot l_{\mathrm{g}}}{2 \sqrt{2}} \cdot \sqrt{\frac{q \cdot N_{\mathrm{d}} \cdot \varepsilon}{V_{\mathrm{bi}}-V_{\mathrm{gs}}^{\prime}}}
$$

Après pincement du canal $\left(V_{\mathrm{gs}}^{\prime}<V_{\mathrm{t}}-0,15 \mathrm{~V}\right)$

$$
C_{\mathrm{gs}}=\varepsilon w \cdot \tan ^{-1} \sqrt{\frac{V_{\mathrm{bi}}-V_{\mathrm{t}}}{V_{\mathrm{t}}-V_{\mathrm{gs}}^{\prime}}}
$$

D'une façon similaire les variations de $C_{\mathrm{gd}}$ sont obtenues en utilisant $\left(V_{\mathrm{g}}^{\prime}-V_{\mathrm{d} 2}\right)$ comme tension de commande [25].

Dans la zone de transition $\left[V_{\mathrm{t}}-0,15 \mathrm{~V}, V_{\mathrm{t}}+\right.$ $0,15 \mathrm{~V}]$ la capacité $C_{\mathrm{gs}}$ est estimée linéairement entre les deux valeurs limites obtenues avec $V_{\mathrm{gs}}^{\prime}=$ $V_{\mathrm{t}}+0,15 \mathrm{~V}$ dans l'équation (6) et $V_{\mathrm{gs}}^{\prime}=$ $V_{\mathrm{t}}-0,15 \mathrm{~V}$ dans l'équation (7).

La figure 6 propose une comparaison entre les capacités $C_{\mathrm{gs}}$ modélisée et mesurée [15] dans le cas du transistor NEC388. L'accord apparaît satisfaisant particulièrement si l'on prend soin d'adjoindre la capacité propre due aux métallisations de grille et de source $C_{\mathrm{bs}}$ (voir paragraphe 2.3.2).

Lors de l'analyse physique du fonctionnement interne du transistor Shur [1] a envisagé une capacité intrinsèque entre source et drain appelée «capacité de domaine ». Cet élément trouve son origine dans l'accumulation des porteurs à l'extrémité drain du canal engendrée par la saturation de la mobilité en 


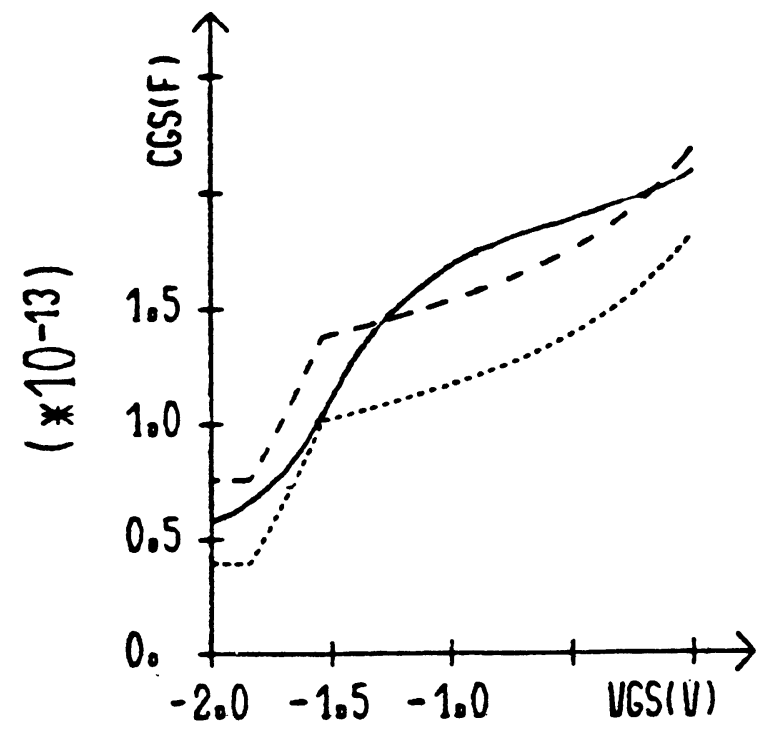

Fig. 6. - Dépendance non linéaire de $C_{\mathrm{gs}}$ pour le NEC388. — résultats expérimentaux, ...... modèle de capacité pour $C_{\mathrm{gs}}$, ------ modèle de capacité pour $C_{\mathrm{gs}}$ avec la capacité interélectrodes ajoutée.

[Capacitance voltage dependence of the NEC388 microwave MESFET. - experimental results, ...... $C_{\mathrm{gs}}$ capacitance model, ------ $C_{\mathrm{gs}}$ capacitance model with proper capacitance of electrodes $C_{\mathrm{bs}}$ added.]

fort champ électrique [26]. Les nouvelles générations de MESFET possèdent des profondeurs typiques de couche active très faibles qui réduisent très fortement la formation du domaine Gunn à l'extrémité drain. Ainsi la capacité de domaine peut être négligée comparée aux capacités propres des métallisations.

2.2.3 La résistance de canal. - En régime de saturation le canal présente deux résistances associées respectivement à la zone validant les hypothèses de Shockley (Fig. 3, zone I) et à la zone de saturation de vitesse (Fig. 3, zone II) [6]. Du fait de la saturation la composante de $R_{\mathrm{i}}$ de la zone II est très importante et elle introduit avec $C_{\mathrm{gs}}$ une constante de temps de grande valeur qui n'influe pas sur le régime transitoire. La composante de $R_{\mathrm{i}}$ issue de la zone de Shockley traduit conjointement avec $C_{\mathrm{gs}}$ le temps et l'énergie nécessaire à la modification de la zone déplétée avant d'injecter de nouveaux porteurs dans la zone de saturation. Cette résistance peut alors être calculée par [15] :

$R_{\mathrm{i}}=\frac{1}{3} \cdot \frac{\text { Tension aux bornes de la zone de Shockley }}{I_{\mathrm{d}}}$.

De façon évidente d'après l'équation (8), $R_{\mathrm{i}}$ est fonction de la polarisation, cependant pour des raisons de simplification liées à nos impératifs de faible coût CPU, nous l'avons choisie constante. Les MESFET des circuits logiques travaillent en majorité en régime de saturation, aussi la détermination de $R_{\mathrm{i}}$ est faite en considérant ce mode de fonctionnement particulier. En utilisant notre modélisation de $I_{\mathrm{d}}$, il existe une polarisation particulière du transistor où le canal vérífie intégralement les hypothèses de Shockley tout en étant juste à la limite de la saturation. Cette polarisation est : $V_{\mathrm{ds}}=0,4 \mathrm{~V}$, $V_{\mathrm{gs}}^{\prime}-V_{\mathrm{t}}=0,4 \mathrm{~V}$. Alors $I_{\mathrm{d}}$ est donnée par l'équation (1) et $R_{\mathrm{i}}$ s'évalue par :

$$
R_{\mathrm{i}}=\frac{1}{1,2 \beta}=\frac{l_{\mathrm{g}} \cdot a_{0}}{0,6 \mu_{0} \cdot w \cdot \varepsilon} .
$$

Nous avons montré [27] par comparaison avec les calculs publiés par Cappy [15] que cette évaluation de $R_{\mathrm{i}}$ est très raisonnable à la polarisation que nous considérons. Bien que nous ne tenions pas compte de la variation de $R_{\mathrm{i}}$ en fonction de $V_{\mathrm{gs}}$ et $V_{\mathrm{ds}}$, ce qui nécessiterait d'évaluer le recul du point de saturation sous la grille, l'équation (9) donne des résultats satisfaisants pour les comportements dynamiques des MESFET (cf. paragraphe 3) et en particulier en ce qui concerne les temps de propagation des oscillateurs en anneau.

2.2.4 Les diodes Schottky. - La simulation grand signal du MESFET impose de prendre en compte les fuites de courant provoquées par le contact Schottky de grille dès que $V_{\mathrm{gs}}>0,5 \mathrm{~V}$. Ce cas est très fréquent lorsque l'on considère les logiques ultrarapides AsGa aussi bien à enrichissement qu'à déplétion. Typiquement les logiques BFL les plus rapides utilisent la limitation d'excursion logique provoquée par la conduction de la diode Schottky grille-source des transistors d'entrée des portes afin d'accroître la rapidité. De même dans le cas de la logique DCFL, le niveau logique haut correspond usuellement à des tensions $V_{\mathrm{gs}}$ de l'ordre de $0,8 \mathrm{~V}$ ce qui entraîne une conduction Schottky.

Une modélisation simple de la conduction Schottky a été résumée par Sze [28]. La source de courant associée à $D 1$ se calcule par:

$$
\begin{aligned}
I_{\mathrm{gs}}=A^{*} \cdot T^{2} \cdot \exp & \left(\frac{q \cdot \phi_{\mathrm{s}}}{k \cdot T}\right) \cdot \frac{w \cdot l_{\mathrm{g}}}{2} \times \\
& \times\left\{\exp \left(\frac{q \cdot V_{\mathrm{gs}}}{N \cdot k \cdot T}\right)-1\right\}
\end{aligned}
$$

Une valeur typique de la constante de Richardson pour l'AsGa est $A^{*}=8,2 \mathrm{~A} / \mathrm{cm}^{2} / \mathrm{K}^{2}$. L'équation (10) s'applique par dualité à $D 2$ en substituant $I_{\mathrm{gs}}$ et $V_{\mathrm{gs}}$ par $I_{\mathrm{gd}}$ et $V_{\mathrm{gd}}$.

\subsection{LES PARAMÈTRES DU TRANSISTOR EXTRINSÈ- QUE.}

2.3.1 Les résistances d'accès. - Des procédures expérimentales existent pour la détermination précise des résistances d'accès des transistors hyperfré- 
quence [29] et sont appliquées ici pour le MESFET TH1408. Dans le cas d'une technologie figée, des formules analytiques peuvent être données, nous avons suivi cette approche pour les MESFET de circuits logiques.

Lorsque le processus technologique est très bien connu les résistances carré des métallisations ainsi que le facteur de contact associé aux contact ohmiques $f_{\mathrm{c}}$ sont parfaitement déterminés. Les équations (11) à (13) permettent alors le calcul des résistances d'accès des MESFET pour chaque jeu de paramètres géométriques

$$
R_{\mathrm{g}}=R_{\square \mathrm{g}} \cdot \frac{w}{l_{\mathrm{g}} \cdot m_{\mathrm{g}}}
$$

$m_{\mathrm{g}}$ tient compte de la résistance distribuée de la grille, il fut introduit pour la première fois par Wolf [30] et une valeur usuelle est $m_{\mathrm{g}}=3$

$$
\begin{aligned}
& R_{\mathrm{s}}=\frac{1}{w} \cdot\left\{\sqrt{f_{\mathrm{c}} \cdot R_{\square_{0}}}+l_{\mathrm{sg}} \cdot R_{\square}\right\} \\
& R_{\mathrm{d}}=\frac{1}{w} \cdot\left\{\sqrt{f_{\mathrm{c}} \cdot R_{\square_{0}}}+l_{\mathrm{gd}} \cdot R_{\square}\right\}
\end{aligned}
$$

Où $R_{\square_{0}}$ est la résistance de couche sous le contact ohmique, et $R_{\square}$ est la résistance de couche dans les espaces entre métallisations

$$
\begin{aligned}
R_{\square_{0}} & =\frac{1}{q \cdot N_{\mathrm{d}} \cdot a_{0} \cdot \mu_{0}} \\
R_{\square} & =\frac{1}{q \cdot N_{\mathrm{d}} \cdot \mu_{0}\left(a_{0}-\sqrt{\frac{2 \cdot \varepsilon \cdot \psi_{\mathrm{ss}}}{q \cdot N_{\mathrm{d}}}}\right)}
\end{aligned}
$$

2.3.2 Les capacités d'électrodes. - Pour évaluer les capacités inter-métallisations nous avons fait appel à la théorie des lignes coplanaires hyperfréquences. La capacité entre deux lignes coplanaires de longueur $w$ sur un substrat d'AsGa est calculée par [31]

$$
C=\varepsilon_{0} \cdot\left(\varepsilon_{\mathrm{r}}+1\right) \cdot w \cdot \frac{K\left(s^{\prime}\right)}{K(s)}
$$

Où $s^{\prime}=\sqrt{1-s^{2}}$, et où le rapport $K\left(s^{\prime}\right) / K(s)$ est défini par

$$
\frac{K\left(s^{\prime}\right)}{K(s)}=\left\{\begin{array}{l}
\frac{1}{\pi} \cdot \log \left(2 \cdot \frac{1+\sqrt{s^{\prime}}}{1-\sqrt{s^{\prime}}}\right) 0<s<\frac{1}{\sqrt{2}} \\
{\left[\frac{1}{\pi} \cdot \log \left(2 \cdot \frac{1+\sqrt{s}}{1-\sqrt{s}}\right)\right]^{-1} \frac{1}{\sqrt{2}}<s<1}
\end{array}\right.
$$

La valeur du paramètre $s$ à utiliser pour le calcul de $C_{\mathrm{bd}}, C_{\mathrm{bs}}$ et $C_{\mathrm{ds}}$ à l'aide des équations (14) et (15) est donnée par les expressions suivantes :

$$
s\left(C_{\mathrm{bd}}\right)=\sqrt{\frac{l_{\mathrm{gd}}}{l_{\mathrm{g}}+l_{\mathrm{gd}}}}
$$

$$
\begin{aligned}
& s\left(C_{\mathrm{bs}}\right)=\sqrt{\frac{l_{\mathrm{gs}}}{l_{\mathrm{g}}+l_{\mathrm{gs}}}} \\
& s\left(C_{\mathrm{ds}}\right)=\frac{\sqrt{2 \cdot l_{\mathrm{sd}} \cdot\left(L+l_{\mathrm{sd}}\right)}}{L+l_{\mathrm{sd}}} .
\end{aligned}
$$

Les paramètres géométriques $\left(L, l_{\mathrm{sg}}, l_{\mathrm{gd}}, l_{\mathrm{sd}}\right.$, $l_{g}$ ) peuvent être obtenus par des mesures optiques sur la puce s'ils ne sont pas fournis par le constructeur.

'L'obtention de ces capacités peut se faire par des méthodes plus rigoureuses, mais aussi beaucoup plus coûteuses en temps de calcul, qui consistent en une résolution complète du problème électromagnétique appliqué à chaque cas particulier de géométrie [32]. Notamment il s'agit-là du seul moyen satisfaisant de tenir compte de l'épaisseur de métallisation des électrodes et en particulier de la grille pour laquelle le type de modélisation que nous proposons ne donnera que des résultats approchés. En pratique l'ordre de grandeur des capacités donné par l'équation (14) est raisonnable, une modélisation poussée n'apporterait que des corrections du second ordre sur les comportements dynamiques des MESFET.

\section{Comparaisons mettant en auvre des comporte- ments dynamiques.}

Le comportement dynamique des MESFET est estimé en régime petit signal à l'aide de mesures de paramètres $S$ hyperfréquence. Aussi avons-nous comparé les paramètres $S$ mesurés et simulés pour le MESFET TH1408 à la polarisation $V_{\mathrm{ds}}=3 \mathrm{~V}$, $V_{\mathrm{gs}}=-1 \mathrm{~V}$ et dans la bande de fréquence 2$18 \mathrm{GHz}$. Ce type de comparaison est très sévère pour un modèle analytique simple voué à la simulation de circuits logiques, en aucun cas les résultats obtenus ne peuvent être comparés à ceux produits par des modélisations hyperfréquences fittées. Cependant ce test est un excellent moyen de montrer la cohérence globale des éléments. Les paramètres $S$ de transmission $S_{21}$ et $S_{12}$ sont représentés sur la partie supérieure de la figure 7. Il apparaît que les phases déduites du modèle sont bonnes alors que les modules sont surévalués dans les deux cas. $S_{21}$ est trop fort à cause de la différence entre la transconductance mesurée en régime statique et celle accessible à travers des paramètres $S$ hyperfréquences. La surévaluation de $S_{12}$ est due pour sa part à une imprécision dans l'évaluation de $C_{\mathrm{gd}}$ en utilisant le modèle donné en [25]. Le paramètre de réflexion $S_{11}$ montre un accord excellent avec la mesure, ce qui corrobore une très bonne modélisation de l'entrée du MESFET $\left(C_{\mathrm{gs}}, R_{\mathrm{i}}\right)$. La différence maximum observable sur $S_{22}$ concerne une rotation de phase trop importante probablement due à la surestimation de $C_{\mathrm{gd}}$.

La simulation d'un circuit logique même à petit 


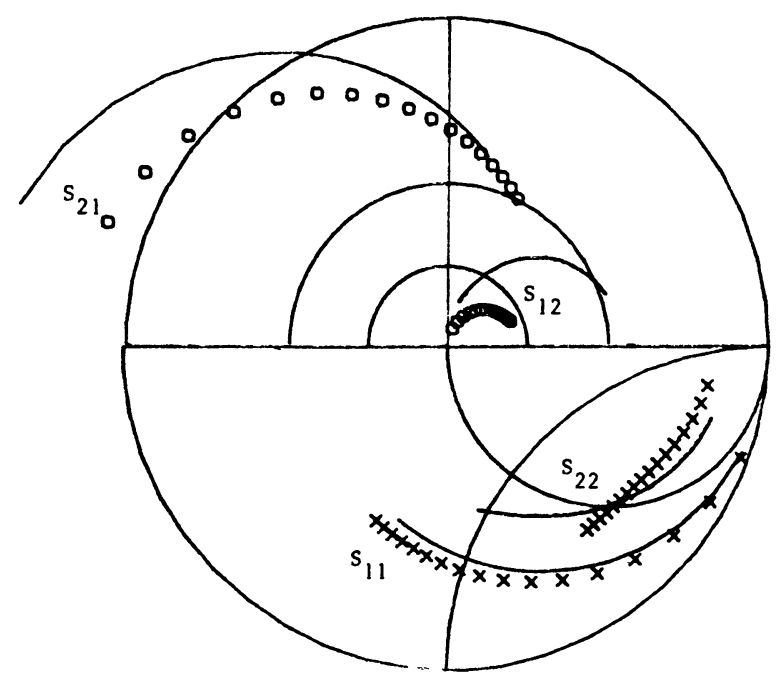

Fig. 7. - Paramètres $S$ du transistor TH1408 à la polarisation $V_{\mathrm{ds}}=3 \mathrm{~V}, V_{\mathrm{gs}}=-1 \mathrm{~V}$. - présent modèle, $\square \square$ et $\times \times \times$ sont les points de mesure des paramètres de transmission $\left(S_{21}, S_{12}\right)$ et de réflexion $\left(S_{11}, S_{22}\right)$ respectivement. Pour une plus grande lisibilité un facteur d'échelle de 2 est appliqué à $S_{12}$ et un facteur de $1 / 2$ à $S_{21}$.

[ $S$ parameters of TH1408 at bias $V_{\mathrm{ds}}=3 \mathrm{~V}, V_{\mathrm{gs}}=-1 \mathrm{~V}$. this model, $\square \square \square$ and $\times \times \times$ are measurement points of transmitted parameters $\left(S_{21}, S_{12}\right)$ and reflected parameters $\left(S_{11}, S_{22}\right)$ respectively. For convenience $S_{21}$ is scaled by a factor 2 and $S_{12}$ by a factor 0.5 .]

niveau d'intégration est un excellent véhicule de test pour montrer la cohérence globale d'un modèle. Un circuit classique d'évaluation des performances d'une technologie est l'oscillateur en anneau. De tels oscillateurs à 55 étages ont été réalisés à Thomson Semiconducteurs DHM/DAG en utilisant une logique BFL implantée de longueur de grille $1 \mu \mathrm{m}$ (voir les paramètres Tab. II). Un temps de propagation moyen par porte logique de 120 ps et une excursion logique de $1 \mathrm{~V}$ ont été mesurés avec un choix de tensions d'alimentation de $+2,5 /-2,5 \mathrm{~V}$. La simulation d'un oscillateur en anneau à 5 étages employant les même paramètres technologiques est donnée figure 8. Nous avons tenu compte dans cet exemple des perturbations introduites par les lignes de propagation entre portes, ceci étant connu comme une source de divergence possible [33]. Dans ce cadre la simulation donne aussi une excursion logique de $1 \mathrm{~V}$ avec un temps de propagation par porte élémentaire de $125 \mathrm{ps,} \mathrm{soit} \mathrm{un} \mathrm{écart} \mathrm{de} 4 \%$ seulement avec la mesure. Cet excellent accord prouve la bonne adéquation du modèle à la simulation de circuits logiques complexes et rapides.

Ce paragraphe donne les performances en temps calcul mesurées sur un ordinateur IBM 3081 afin de montrer la capacité du modèle à simuler des circuits MSI voire LSI. Le calcul d'un MESFET élémentaire par pas de calcul du simulateur temporel est de $\sim 100 \mu \mathrm{s}$. Avec le simulateur temporel MACPRO

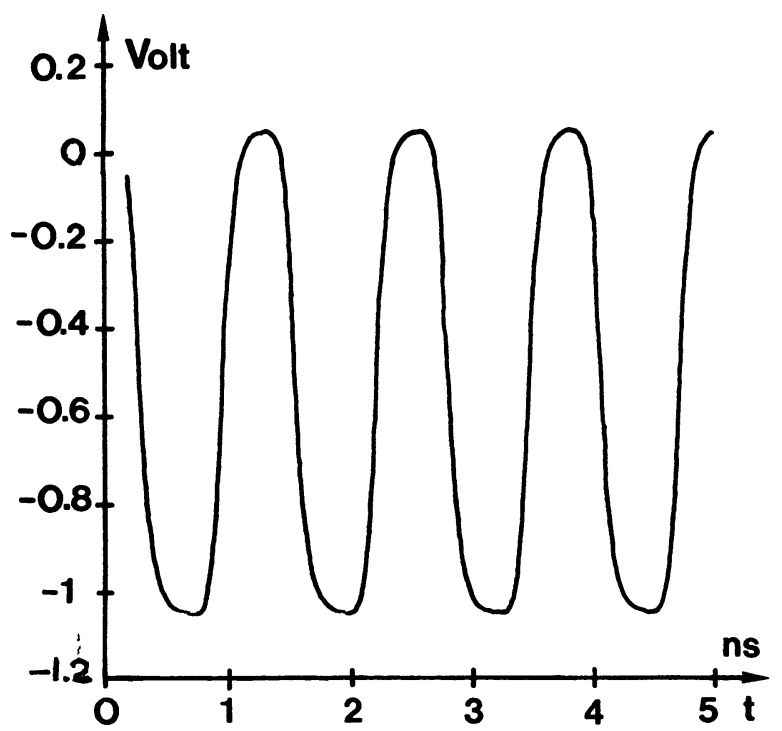

Fig. 8. - Simulation d'un oscillateur en anneau à 5 étages. Le temps de propagation par porte est de 125 ps dans la simulation alors qu'il est de 120 ps dans la mesure' des oscillateurs en anneau à 55 étages.

[BFL 5-stage ring oscillator simulation. The propagation delay time of 125 ps per gate found in this simulation has to be compared to the average 120 ps found in experimental realizations of 55-stage ring oscillators.]

[34] le coût attendu pour le calcul d'un circuit MSI de 100 portes BFL est de $180 \mathrm{~s}$ sur 5000 pas de calcul. L'estimation précédente n'inclue pas les effets propres à la latence qui sont traités dans le simulateur, et qui réduiraient d'un facteur au moins 2 le coût précédent suivant le nombre de portes logiques inactives à chaque pas de calcul.

\section{Influence des paramètres du modèle sur la réponse dynamique des portes BFL.}

Le modèle que nous avons développé a été utilisé pour simuler des portes logiques BFL correspondant à une technologie projetée par Thomson-DHM (voir Tab. II). Ces caractéristiques principales sont les suivantes.

i) La grille submicronique $(0,6 \mu \mathrm{m})$ est déposée après creusement de $600 \AA$ de la couche active afin d'obtenir la tension de seuil choisie $\left(V_{\mathrm{t}}=-1,2 \mathrm{~V}\right)$ tout en conservant des résistances d'accès à une faible valeur.

ii) Deux diodes Schottky seulement sont utilisées dans la conception de la porte BFL afin de réduire l'excursion logique par la conduction de la jonction grille-source des transistors d'entrée. La vitesse de commutation est accrue ainsi que la puissance dissipée.

L'étude de l'influence des variations des paramètres du modèle sur le régime transitoire des portes BFL est effectuée en considérant successivement les 
paramètres du transistor intrinsèque et extrinsèque. Le circuit utilisé est donné sur la figure 9 avec les largeurs de chacun des transistors, il ne fait appel qu'à une seule commutation de porte. La diode Schottky chargeant la porte simule l'entrée de l'inverseur suivant, et les temps de montée et de descente de la commande appliquée sont de 50 ps.

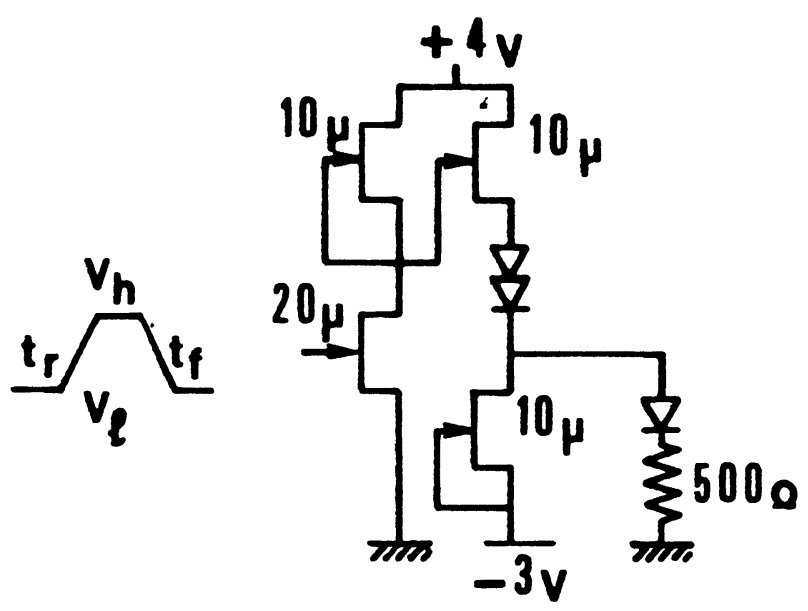

Fig. 9. - Circuit simulé pour l'étude de l'influence des paramètres du modèle sur la réponse dynamique des portes BFL.

[Circuit schematic used to study the influence of the model parameters upon the dynamic response of BFL gates.]

Comme la principale différence de ce travail concerne la modélisation de la source de courant interne du transistor, nous avons simulé une commutation ultra-rapide d'une porte BFL avec deux descriptions possibles de $I_{\mathrm{d}}$. Les figures $10 \mathrm{a}$ et $10 \mathrm{~b}$ montrent les deux caractéristiques $I-V$ internes qui correspondent :

i) au modèle développé pour le simulateur CIRCEC qui utilise une formulation relativement compliquée de la source de courant issue du modèle de Shockley et qui tient compte de la variation de mobilité des porteurs par une formule analytique [35] ;

ii) au modèle développé dans ce travail.

Malgré une différence notable sur les valeurs de $I_{\mathrm{d}}$ en saturation (30\% à $\left.I_{\mathrm{dss}}\right)$ et sur la forme de $I_{\mathrm{d}}$ notamment à faible $V_{\mathrm{ds}}$, les écarts principaux entre les simulations temporelles (Fig. 10c) sont un décalage des niveaux logiques de $90 \mathrm{mV}$ seulement alors que les temps de montée et de descente sont comparables. Trois raisons justifient ce résultat.

i) Le point de basculement de cette porte BFL est à $V_{\mathrm{gs}} \simeq-0,6 \mathrm{~V}$, là où la différence entre les caractéristiques est nettement plus faible.

ii) Le basculement de l'état haut à l'état bas d'une porte BFL est avant tout dicté par l'arrêt de la

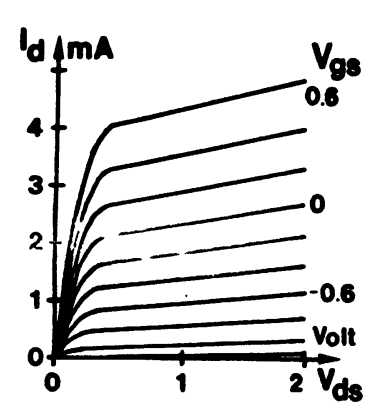

a)

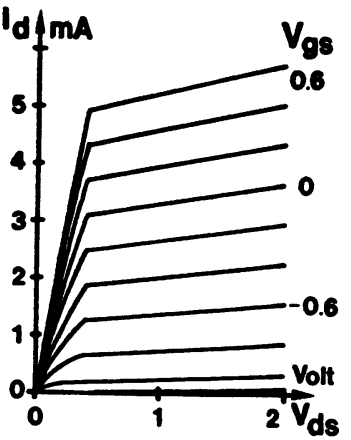

b)

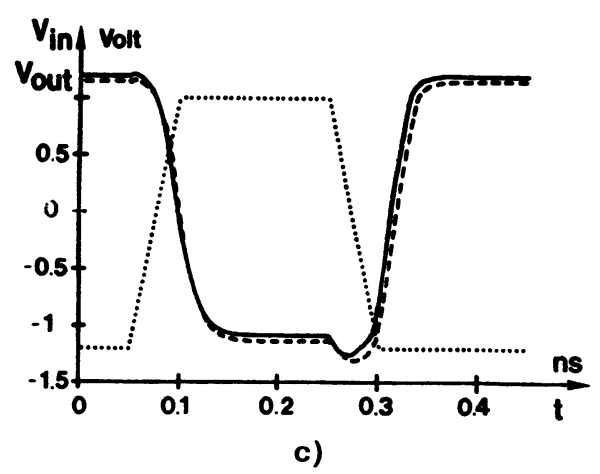

Fig. 10. - Influence de la description de la caractéristique $I-V$ sur le régime transitoire d'une porte BFL. (a) Caractéristique interne du présent modèle. (b) Caractéristique interne du modèle CIRCEC. (c) Comparaison des comportements dynamiques avec les deux caractéristiques précédentes (a) et (b). ...... tension de commande, -.---ce modèle, $\longrightarrow$ modèle CIRCEC.

[Influence of the $I-V$ characteristic on the dynamic response of a BFL gate. (a) Internal characteristic of the present model. (b) Internal characteristic of the CIRCEC model. (c) Compared dynamic response of the BFL gate with the $I-V$ characteristics (a) and (b). ...... input voltage, ----- this work, CIRCEC model.]

conduction des diodes Schottky de décalage du buffer de sortie de la porte.

iii) Le comportement dynamique des portes dépend fortement de l'ensemble des éléments parasites comme le montre l'étude suivante sur l'influence des paramètres du modèle.

L'ensemble des résultats de l'étude de l'influence des paramètres du modèle sur le régime dynamique des portes BFL est résumé tableau III. Dans le détail, les remarques suivantes s'imposent.

i) La variation des paramètres intrinsèques du modèle $\mu_{0}$ et $l_{\mathrm{g}}$ affecte essentiellement $\beta$ et $R_{\mathrm{i}}$. Il en découle une variation du gain et du retard au délenchement de la porte logique qui vont dans le même sens. Lorsque $l_{\mathrm{g}}$ diminue (Fig. 11), la commutation est de plus en plus rapide. De même une augmentation de $\mu_{0}$ (Fig. 12) produit un résultat analogue avec des temps de commutation de plus en 
Tableau III. - Influence des paramètres du modèle sur la réponse dynamique d'une porte BFL. Les chiffres romains sont relatifs à la discussion $d u$ paragraphe 4.

[Influence of technological parameter variation on characteristic dynamic risetime and falltime of a BFL gate. The roman numbers are related to the discussion of section 4.]

\begin{tabular}{|c|c|c|c|}
\hline & & $\begin{array}{l}\text { Temps de } \\
\text { Montée } \\
\text { (ps) }\end{array}$ & $\begin{array}{l}\text { Temps de } \\
\text { Descente } \\
\text { (ps) }\end{array}$ \\
\hline \multicolumn{2}{|c|}{$\begin{array}{l}\text { Tous les parametres a leur valeur } \\
\text { nominale (Table 2, BFL projetée) }\end{array}$} & 32 & 47 \\
\hline $\begin{array}{l}\text { Tous nominaux } \\
\text { sauf }\end{array}$ & $\begin{array}{l}\text { Valeur du } \\
\text { parametre }\end{array}$ & & \\
\hline$\underset{\substack{\mu_{0} \\
\mu_{0} \\
l g}}{1 \mathrm{~g}}$ & \begin{tabular}{|ccc}
$2000 \mathrm{~cm}^{2} / \mathrm{v} / \mathrm{s}$ & $(-25 \%)$ \\
$3000 \mathrm{~cm}^{2} / \mathrm{v} / \mathrm{s}$ & $(+25 \%)$ \\
$0.8 \mu \mathrm{m}$ & $(+33 \%)$ \\
$0.4 \mu \mathrm{m}$ & $(-33 \%)$
\end{tabular} & $\begin{array}{ll}38 & (+19 \%) \\
30 & (-6 \%) \\
38 & (+19 \%) \\
22 & (-31 \%)\end{array}$ & $\begin{array}{l}52(+11 \%) \\
39(-17 \%) \\
50(+6 \%) \\
33(-30 \%)\end{array}$ \\
\hline (ii) Cgs, Cgd (*) & $10 \mathrm{fF}$ pour $w=20 \mu \mathrm{m}$ & $53(+66 \%)$ & $55(+17 \%)$ \\
\hline $\begin{array}{lll} & \text { Rg } & \\
\text { (iii) } & \text { Rs } & (* *) \\
& \text { Rd } & (* *)\end{array}$ & $\begin{array}{l}0 \\
0 \\
0\end{array}$ & $\begin{array}{ll}32 & (0 \%) \\
32 & (0 \%) \\
32 & (0 \%)\end{array}$ & $\begin{array}{l}47(0 \%) \\
43 \quad(-9 \%) \\
47(0 \%)\end{array}$ \\
\hline (iv) Cds,Cbs, Cbd & 0 & $25(-22 \%)$ & $45(-4 \%)$ \\
\hline
\end{tabular}

(*) Cette valeur a été choisie au milieu de la variation de Cgs.

(**) L'excursion logique devient plus grande de $0,1 \mathrm{~V}$ dans ce cas. Malgré une pente $\mathrm{d} V / \mathrm{d} t$ du signal plus grande, les temps caractéristiques de la porte ne sont pas vraiment modifiés.

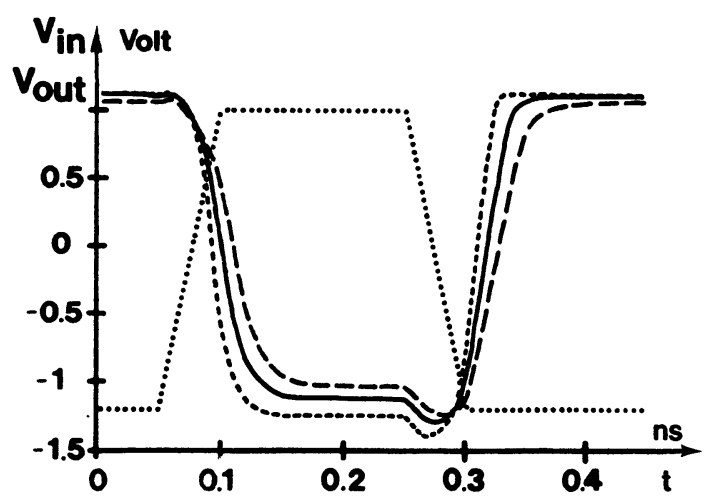

Fig. 11. - Influence de la longueur de grille sur la réponse temporelle des portes BFL...... $l_{\mathrm{g}}=0,4 \mu \mathrm{m},-$ $l_{\mathrm{g}}=0.6 \mu \mathrm{m}, \cdots l_{\mathrm{g}}=0,8 \mu \mathrm{m}$.

[Gatelength influence on dynamic time response of BFL gates. $\quad \ldots \ldots . l_{\mathrm{g}}=0.4 \mu \mathrm{m}, \quad l_{\mathrm{g}}=0.6 \mu \mathrm{m}$, -.--$l_{\mathrm{g}}=0.8 \mu \mathrm{m}$.

plus courts. Ces deux paramètres doivent être connus avec précision ou ajustés en fonction de résultats de mesure. Nous avons déjà soulevé le problème de la détermination de $\mu_{0}$ sous la grille (paragraphe 2.2.1), pour ce qui concerne une éventuelle indétermination sur $l_{\mathrm{g}}$ le problème se résout aisément par des mesures optiques sur le circuit puisque cette dimension est dépendante du processus technologique et invariante sur la plaquette.

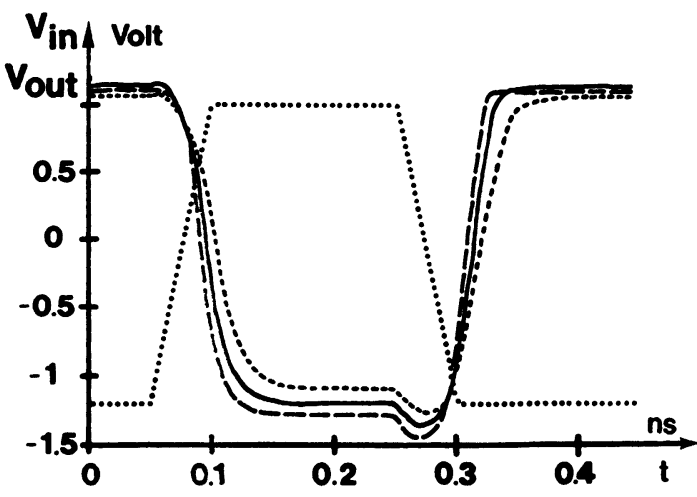

Fig. 12. - Influence de la valeur de mobilité en champ faible sur la réponse temporelle des portes BFL. ...... $\mu_{0}=2000 \mathrm{~cm}^{2} / \mathrm{V} \cdot \mathrm{s}, \longrightarrow \mu_{0}=3000 \mathrm{~cm}^{2} / \mathrm{V} \cdot \mathrm{s}$, $\mu_{0}=4000 \mathrm{~cm}^{2} / \mathrm{V} \cdot \mathrm{s}$.

[Low-field mobility influence on dynamic time response of BFL gates. …. $\mu_{0}=2000 \mathrm{~cm}^{2} / \mathrm{V} \cdot \mathrm{s}$, $\mu_{0}=3000 \mathrm{~cm}^{2} / \mathrm{V} \cdot \mathrm{s},-\cdots \mu_{0}=4000 \mathrm{~cm}^{2} / \mathrm{V} \cdot \mathrm{s}$.]

ii) Les dépendances non linéaires des capacités intrinsèques du MESFET sont une des clés de la description correcte du régime transitoire. Si ces capacités sont supposées constantes, l'asymétrie originale que l'on observe entre temps de montée et temps de descente du signal disparaît, modifiant ainsi sensiblement les temps caractéristiques de la porte BFL.

iii) La résistance de grille qui possède une très grande importance dans le régime petit signal hyperfréquence n'a presque aucune incidence sur la forme du signal de commutation. La raison peut être trouvée en comparant sa valeur $\left(R_{\mathrm{g}}=17 \Omega\right)$ à celle de $R_{\mathrm{i}}\left(R_{\mathrm{i}}=215 \Omega\right)$ pour un MESFET typique de circuit logique de largeur $w=20 \mu \mathrm{m}$. Par contre les résistances de drain et de source ne peuvent être négligées à cause de la contre-réaction (résistance de source) et de la chute de tension (résistance de drain) qu'elles produisent. L'influence de la résistance de source est représentée sur la figure 13. Le seul écart se situe dans une modification de l'excursion logique alors que les temps caractéristiques de la porte logique ne sont pas modifiés.

iv) L'importance des capacités du modèle est illustrée par la figure 14. Lors de la commutation de l'état bas à l'état haut le MESFET d'entrée de la porte travaille très près du pincement. Le temps de montée est alors influencé essentiellement par les capacités d'entrée du transistor $\left(C_{\mathrm{gs}}\right.$ et $\left.C_{\mathrm{bs}}\right)$ et par la capacité drain-source $C_{\mathrm{ds}}$ qui limite l'accroissement de $V_{\mathrm{ds}}$. Si les capacités inter-électrodes sont négligées, ce temps de montée est sous-évalué. Lors de la commutation de l'état haut à l'état bas aucun effet notable n'est observable parce que le temps de descente est défini par l'arrêt de la conduction des 


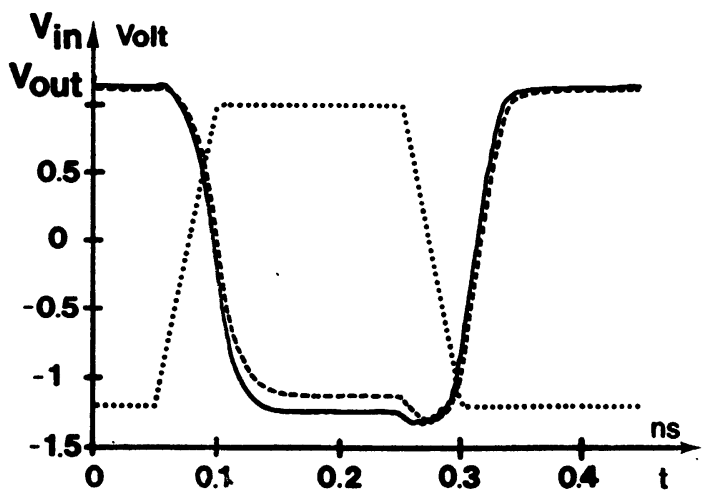

Fig. 13. - Influence de la résistance de source sur la réponse temporelle des portes BFL. - $R_{\mathrm{s}}=0 \Omega$, --.-$R_{\mathrm{s}}$ à la valeur nominale (Tab. II).

[Influence of the source resistance on dynamic time response of BFL gate. $-R_{\mathrm{s}}=0 \Omega,--R_{\mathrm{s}}$ to nominal value of (Tab. II).]

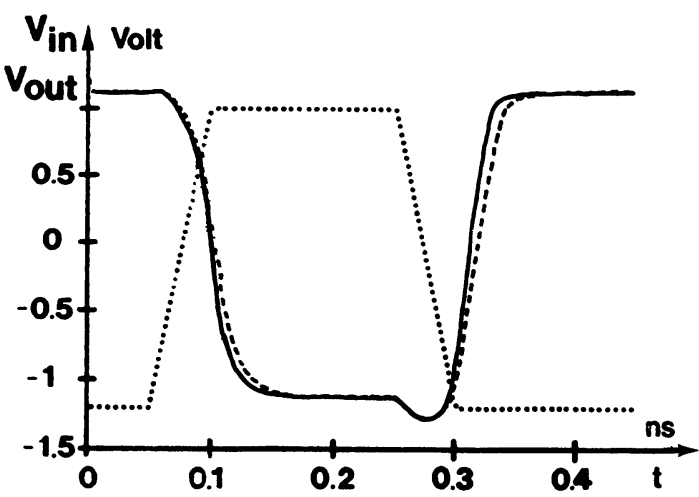

Fig. 14. - Influence des capacités inter-électrodes sur la réponse temporelle des portes BFL. $-C_{\mathrm{bs}}, C_{\mathrm{bd}}$ et $C_{\mathrm{ds}}$ nulles, ----- $C_{\mathrm{bs}}, C_{\mathrm{bd}}$ et $C_{\mathrm{ds}}$ aux valeurs nominales (Tab. II).

[Influence of the electrodes capacitances on dynamic time response of BFL gate. $-C_{\mathrm{bs}}, C_{\mathrm{bd}}$ and $C_{\mathrm{ds}}$ set to zero, -.-- $C_{\mathrm{bs}}, C_{\mathrm{bd}}$ and $C_{\mathrm{ds}}$ set to nominal values (Tab. II).]

diodes de décalage dans l'étage de sortie de la porte logique BFL.

En conclusion, les résultats du tableau III montrent clairement que la dynamique des portes logiques BFL est déterminée principalement par les éléments parasites du MESFET. Notre modèle a été récemment appliqué à la simulation de circuits logiques en technologie DCFL [36] et l'ensemble des remarques précédentes est valable avec cette autre topologie de la porte élémentaire.

\section{Conclusion.}

Un modèle analytique du MESFET AsGa voué à la simulation de circuits logiques a été complètement décrit. Le compromis entre la précision requise par les simulations temporelles et un faible coût CPU autorisant la prédiction du fonctionnement de circuits à fort niveau d'intégration nous a conduit à une formulation simplifiée de la source de courant interne du transistor. Des comparaisons expérimentales vis-à-vis de caractéristiques $I-V$ de transistors hyperfréquence ont cependant montré un accord satisfaisant.

Un oscillateur en anneau en technologie BFL a été simulé et il montre une différence de $4 \%$ seulement avec les résultats de mesure. Une comparaison avec des paramètres $S$ d'un MESFET hyperfréquence qui est très sévère pour un modèle destiné aux circuits logiques, a montré un comportement d'ensemble cohérent même s'il ne peut être comparé à celui accessible avec un modèle petit signal hyperfréquence.

Une étude de sensibilité des paramètres du modèle sur la réponse dynamique de portes logiques BFL est proposée. Un résultat assez surprenant est démontré : la description de la caractéristique autant pour ce qui concerne sa forme que pour les niveaux de courant de saturation, a peu d'influence sur le régime transitoire des portes. Par contre les capacités tant intrinsèques qu'extrinsèques du modèle de MESFET sont primordiales pour une description exacte de la commutation.

La cohérence du modèle et ses possibilités dans le domaine de la simulation de circuit est prouvée. A l'aide de ce modèle des prédictions précises de leurs comportements sont possibles en tenant compte des perturbations apportées par la propagation des signaux de long des lignes et/ou du couplage entre plusieurs lignes à l'intérieur même du circuit intégré.

\section{Remerciements.}

Les auteurs remercient M. le Professeur R. Castagné de l'I.E.F., MM. M. Gloanec et B. Carnez de Thomson-Semiconducteurs DHM/DAG pour les discussions très utiles que nous avons eues, ainsi que pour les mesures du MESFET TH1408 et de l'oscillateur en anneau. 


\section{Bibliographie}

[1] Shur, M. S., Solid-State Electron. 22 (1979) 723.

[2] Gough, R. G., IEEE J. Solid-State Circuits SC-17 (1982) 666.

[3] Curtice, W. R., Yun, Y.-H., IEEE Trans. Electron Devices ED-28 (1981) 954.

[4] Curtice, W. R., CAMisa, R. L., IEEE Trans. Microwave Theor. Tech. MTT-32 (1984) 1573.

[5] Curtice, W. R., Ettenberg, M., IEEE Trans. Microwave Theor. Tech. MTT-33 (1985) 1383.

[6] Boccon-Gibod, D., Acta Electronica 23 (1980) 99.

[7] Curtice, W. R., IEEE Trans. Microwave Theor. Tech. MTT-28 (1980) 448.

[8] Kacprzak, T., Materka, A., IEEE J. Solid-State Circuits SC-18 (1983) 211. Cette référence étant une généralisation de :

TAKI, T., IEEE J. Solid-State Circuits SC-13 (1978) 724.

[9] ChuA, L. O., Sing, Y. W., IEEE Trans. Electron Devices ED-30 (1983) 825.

[10] Sussman-Fort, S. E., NARASIMHan, S., MAYARAM, K., IEEE Trans. Microwave Theor. Tech. MTT-32 (1984) 471.

[11] Golio, J. M., Hauser, J. R., Blakey, P. A., IEEE Circuits Devices Magazine (Sept. 1985) 21.

[12] Sussman-Fort, S. E., Hantgan, J. C., Huang, F. L., IEEE Trans. Microwave Theor. Tech. MTT-34 (1986) 1115.

[13] Murzin, J.-Y., Thèse de Troisième Cycle (Université Paris-Sud) 1984.

[14] Crozat, P., Chusseau, L., Bedouani, M., Zounon, A., AdDE, R., GaAs IC Symposium Digest, Monterey (1985) 191.

[15] CAPPY, A., Thèse de Troisième Cycle (Université de Lille) 1981.

[16] Shockley, W., Proc. IRE 40 (1952) 1365.

[17] Fawcett, W., Boardman, A. D., Swain, S., J. Phys. Chem. Solids 31 (1970) 1963.

[18] Castagné, R., Communication privée.

[19] LeE, K., Shur, M. S., LeE, K., Vu, T. T., Roberts, P. C. T., HeLIX, M. J., IEEE Trans. Electron Devices ED-31 (1984) 390.
[20] Kennedy, D. P., O’Brien, R. R., Electron. Lett. 7 (1971) 714.

[21] Yamaguchi, K., Kodera, H., IEEE Trans. Electron Devices ED-23 (1976) 545.

[22] WadA, T., Frey, J., IEEE J. Solid-State Circuits SC14 (1979) 398. -

[23] Carnez, B., Cappy, A., Kaszynski, A., Constant, E., Salmer, G., J. Appl. Phys. 51 (1980) 784.

[24] Pone, J.-F., Castagné, R., Courat, J.-P., ARNODO, C., IEEE Trans. Electron Devices ED-29 (1982) 1244.

[25] Takada, T., Yokoyama, K., IdA, M., Sudo, T., IEEE Trans. Microwave Theor. Tech. MTT-30 (1982) 719.

[26] Yamaguchi, K., Asai, S., Kodera, H., IEEE Trans. Electron Devices ED-23 (1976) 1283.

[27] Chusseau, L., Thèse de Troisième Cycle (Université Paris-Sud) 1985.

[28] Sze, S. M., Physics of Semiconductors Devices (Second Edition, Wiley) 1981.

[29] Urien, P., Delagebeaudeuf, D., Electron. Lett. 19 (1983) 702.

[30] Wolf, P., IBM J. Res. Dev. 14 (1970) 125.

[31] Gupta, K. C., Garg, R., Chadha, R., ComputerAided Design of Microwave Circuits (Artech House) 1981.

[32] Alexopoulos, N. G., Maupin, J. A., Greiling, P. T., IEEE Trans. Microwave Theor. Tech. MTT-28 (1980) 459.

[33] Chang, C. T. M., Namordi, M. R., White, W. A., IEEE Trans. Electron Devices ED-29 (1982) 1805.

[34] Chusseau, L., Crozat, P., Jewett, R., Adde, R., Journées Nationales Micro-ondes, Lannion, juin 1984.

[35] Bert, G., Kazinsky, A., Peltier, M., Communication privée, Note Interne THOMSONCSF/LCR, No 195/GB/CL.

[36] BedouAnI, M., Communication privée. 